\title{
Linear combinations of partitions of unity with restricted supports
}

\author{
by
}

\author{
Christian Richter (Paris)
}

\begin{abstract}
Given a locally finite open covering $\mathcal{C}$ of a normal space $X$ and a Hausdorff topological vector space $E$, we characterize all continuous functions $f: X \rightarrow E$ which admit a representation $f=\sum_{C \in \mathcal{C}} a_{C} \varphi_{C}$ with $a_{C} \in E$ and a partition of unity $\left\{\varphi_{C}: C \in \mathcal{C}\right\}$ subordinate to $\mathcal{C}$.

As an application, we determine the class of all functions $f \in C(|\mathcal{P}|)$ on the underlying space $|\mathcal{P}|$ of a Euclidean complex $\mathcal{P}$ such that, for each polytope $P \in \mathcal{P}$, the restriction $\left.f\right|_{P}$ attains its extrema at vertices of $P$. Finally, a class of extremal functions on the metric space $\left([-1,1]^{m}, d_{\infty}\right)$ is characterized, which appears in approximation by so-called controllable partitions of unity.
\end{abstract}

1. The representation theorem and first conclusions. Let $X$ be a topological space. A system $\left\{\varphi_{\iota}: \iota \in \mathcal{I}\right\}$ of continuous real-valued functions is called a partition of unity on $X$ if $\varphi_{\iota} \geq 0$ for all $\iota \in \mathcal{I}, \sum_{\iota \in \mathcal{I}} \varphi_{\iota} \equiv 1$, and the open covering $\left\{\varphi_{\iota}^{-1}((0,1]): \iota \in \mathcal{I}\right\}$ is locally finite. For a locally finite open covering $\mathcal{C}=\left\{C_{\iota}: \iota \in \mathcal{I}\right\}$ of $X$, a partition of unity $\left\{\varphi_{\iota}: \iota \in \mathcal{I}\right\}$ is said to be subordinate to $\mathcal{C}$ if $\varphi_{\iota}^{-1}((0,1]) \subseteq C_{\iota}$ for all $\iota \in \mathcal{I}$ (cf. [10], p. 352).

For a fixed locally finite open covering $\mathcal{C}$ of $X$ and for a Hausdorff topological vector space $E$, we want to characterize all continuous functions $f: X \rightarrow E$ which admit a representation $f=\sum_{C \in \mathcal{C}} a_{C} \varphi_{C}$ with vectors $a_{C} \in E$ and a partition of unity $\left\{\varphi_{C}: C \in \mathcal{C}\right\}$ subordinate to $\mathcal{C}$. The following theorem solves this question for normal spaces $X$. We shall see that the claim of this very general theorem can even be used for characterizing normal spaces among $\mathrm{T}_{1}$-spaces. Besides that, we shall present an application concerning continuous real-valued functions on polytopes and on Euclidean complexes in $\mathbb{R}^{m}$ as they appear in optimization theory (see Section 2).

2000 Mathematics Subject Classification: 54D15, 49J99, 52B11, 41A30.

Key words and phrases: partition of unity subordinate to a covering, continuous selection, polyhedral complex, entropy numbers, non-linear approximation.

The research was partially supported by DFG Grant RI 1087/2. 
A further application deals with continuous real-valued functions on compact metric spaces $(X, d)$ and their approximation by linear combinations of partitions of unity related to the entropy numbers of $(X, d)$ (see Section 3 ).

In the theorem we use the symbol $\operatorname{conv}(\cdot)$ for the convex hull.

TheOREM 1. Let $X$ be a normal space, $\mathcal{C}$ a locally finite open covering of $X, E$ a Hausdorff topological vector space, $f: X \rightarrow E$ a continuous function, and $a_{C} \in E$ for $C \in \mathcal{C}$. Then the following are equivalent:

(i) There exists a partition of unity $\left\{\varphi_{C}: C \in \mathcal{C}\right\}$ subordinate to $\mathcal{C}$ such that

(ii) For all $x \in X$,

$$
f=\sum_{C \in \mathcal{C}} a_{C} \varphi_{C}
$$

$$
f(x) \in \operatorname{conv}\left\{a_{C}: x \in C \in \mathcal{C}\right\} .
$$

Proof. The implication (i) $\Rightarrow$ (ii) is evident (cf. [10], p. 354).

For the proof of the converse we assume $\mathcal{C}$ to be well ordered. The partition functions $\varphi_{C_{0}}, C_{0} \in \mathcal{C}$, will be obtained by transfinite induction as continuous selections of set-valued functions $\Phi_{C_{0}}: X \rightarrow 2^{\mathbb{R}}$ using a selection theorem from Michael's paper [5]. The beginning of the induction will be treated in the same way as the inductive steps.

We define

$$
\begin{array}{r}
\Phi_{C_{0}}(x)=\left\{\mu_{C_{0}} \text { : there exist }\left\{\mu_{C} \geq 0\right\}_{C \in \mathcal{C}} \text { such that } \sum_{C \in \mathcal{C}} \mu_{C}=1, \mu_{C}=0\right. \\
\text { if } \left.x \notin C, \sum_{C \in \mathcal{C}} a_{C} \mu_{C}=f(x) \text {, and } \mu_{C}=\varphi_{C}(x) \text { for all } C<C_{0}\right\},
\end{array}
$$

where the functions $\varphi_{C}, C<C_{0}$, are supposed to be already known. Note that for fixed $x$ all but finitely many values $\mu_{C}$ vanish. Obviously, $\Phi_{C_{0}}(x)$ is convex and compact. If $C_{0}$ is the smallest element of $\mathcal{C}$, then $\Phi_{C_{0}}(x) \neq \emptyset$ by assumption (ii). Otherwise one obtains $\Phi_{C_{0}}(x) \neq \emptyset$, because $\Phi_{C}(x) \neq \emptyset$ for all $C<C_{0}$ according to the induction hypothesis. Now Theorem $3.1^{\prime}$ of [5] says that $\Phi_{C_{0}}$ admits a continuous selection $\varphi_{C_{0}}$ if $\Phi_{C_{0}}$ is lower semicontinuous (l.s.c.).

Let $x_{0} \in X$ be fixed. We choose an open neighbourhood $U$ of $x_{0}$ which intersects only finitely many sets from $\mathcal{C}$, say $C_{1}, \ldots, C_{n}$, and which, moreover, satisfies

$$
U \subseteq \bigcap_{x_{0} \in C \in \mathcal{C}} C .
$$

If $C_{0} \notin\left\{C_{1}, \ldots, C_{n}\right\}$ then $\Phi_{C_{0}}$ is l.s.c. at $x_{0}$, since then $\Phi_{C_{0}}(x)=\{0\}$ for all $x \in U$. Now let us assume that $C_{0} \in\left\{C_{1}, \ldots, C_{n}\right\}$, say $C_{1}<\ldots<C_{k+1}=$ $C_{0}<C_{k+2}<\ldots<C_{n}$. We use the subsimplex $S(x)=\left\{\left(\mu_{1}, \ldots, \mu_{n}\right) \in S\right.$ : $\mu_{i}=0$ for $\left.x \notin C_{i}\right\}$ of the simplex $S=\left\{\left(\mu_{1}, \ldots, \mu_{n}\right): \mu_{i} \geq 0, \sum_{i=1}^{n} \mu_{i}=1\right\}$, 
the linear map $\pi\left(\mu_{1}, \ldots, \mu_{n}\right)=\left(\sum_{i=1}^{n} a_{C_{i}} \mu_{i} ; \mu_{1}, \ldots, \mu_{k}\right)$, the continuous map $g(x)=\left(f(x) ; \varphi_{C_{1}}(x), \ldots, \varphi_{C_{k}}(x)\right)$, and the set-valued function

$$
\Psi(x)=\left\{\left(\mu_{1}, \ldots, \mu_{n}\right) \in S(x): \pi\left(\mu_{1}, \ldots, \mu_{n}\right)=g(x)\right\}=S(x) \cap \pi^{-1}(g(x))
$$

in order to rewrite $\Phi_{C_{0}}(x), x \in U$, as

$$
\begin{aligned}
\Phi_{C_{0}}(x)=\left\{\mu_{k+1}: \text { there exists }\left(\mu_{1}, \ldots, \mu_{n}\right)\right. & \in \mathbb{R}^{n} \\
& \text { with } \left.\left(\mu_{1}, \ldots, \mu_{n}\right) \in \Psi(x)\right\} .
\end{aligned}
$$

Now it suffices to show that $\Psi$ is l.s.c. at $x_{0}$, since this obviously implies that $\Phi_{C_{0}}$ is l.s.c. at $x_{0}$.

Let an open set $W \subseteq \mathbb{R}^{n}$ be fixed such that $\Psi\left(x_{0}\right) \cap W \neq \emptyset$. We must find a neighbourhood $V$ of $x_{0}$ such that $\Psi(x) \cap W \neq \emptyset$ for all $x \in V$. For every simplex $S(x)$, the restriction of the linear map $\pi: \mathbb{R}^{n} \rightarrow E \times \mathbb{R}^{k}$ to $S(x)$ is an open map onto its image $\pi(S(x))$. Hence there exists an open set $G_{S(x)} \subseteq E \times \mathbb{R}^{k}$ such that

$$
\pi(S(x) \cap W)=\pi(S(x)) \cap G_{S(x)} .
$$

Moreover, $g\left(x_{0}\right) \in G_{S(x)}$. Indeed, we can choose a vector $\left(\mu_{1}, \ldots, \mu_{n}\right) \in$ $\Psi\left(x_{0}\right) \cap W$. Hence $g\left(x_{0}\right)=\pi\left(\mu_{1}, \ldots, \mu_{n}\right) \in \pi\left(S\left(x_{0}\right) \cap W\right)$ by the definition of $\Psi\left(x_{0}\right)$. The inclusion (1) yields $S\left(x_{0}\right) \subseteq S(x)$ for all $x \in U$. Hence $g\left(x_{0}\right) \in$ $\pi(S(x) \cap W) \subseteq G_{S(x)}$

Since there exist only finitely many simplices $S(x), x \in U$, the set $V=$ $U \cap g^{-1}\left(\bigcap_{x \in U} G_{S(x)}\right)$ is an open neighbourhood of $x_{0}$. For all $x \in V$, we trivially have $g(x) \in G_{S(x)}$ and, moreover, $g(x) \in \pi(S(x))$, for $\Psi(x) \neq \emptyset$. Accordingly, $g(x) \in \pi(S(x)) \cap G_{S(x)}=\pi(S(x) \cap W)$ and finally $\Psi(x) \cap W=$ $S(x) \cap \pi^{-1}(g(x)) \cap W \neq \emptyset$. Hence $\Psi$ is l.s.c. at $x_{0}$.

Now Theorem $3.1^{\prime}$ of [5] yields a continuous selection $\varphi_{C_{0}}$ of $\Phi_{C_{0}}$. It follows from the definition of the multifunctions $\Phi_{C}, C \in \mathcal{C}$, that the resulting functions $\varphi_{C}$ form a partition of unity with the properties claimed in (i).

As a special case we consider peaked coverings $\mathcal{C}$ of $X$. A covering $\mathcal{C}$ is called peaked if, for all $C_{0} \in \mathcal{C}$, the set $C_{0} \backslash \bigcup_{C \in \mathcal{C} \backslash\left\{C_{0}\right\}} C$ is non-empty. All points $x \in C_{0} \backslash \bigcup_{C \in \mathcal{C} \backslash\left\{C_{0}\right\}} C$ are called peaks of the covering set $C_{0}$.

The motivation for this name is given by the concept of a peaked partition of unity $\left\{\varphi_{\iota}: \iota \in \mathcal{I}\right\}$ introduced in [6], which is defined by the property that, for all $\iota \in \mathcal{I}$, there exists a point $x \in X$ with $\varphi_{\iota}(x)=1$. Clearly, a peaked partition of unity gives rise to a peaked covering $\left\{\varphi_{\iota}^{-1}((0,1]): \iota \in \mathcal{I}\right\}$. Conversely, any partition of unity $\left\{\varphi_{\iota}: \iota \in \mathcal{I}\right\}$ subordinate to a peaked covering $\left\{C_{\iota}: \iota \in \mathcal{I}\right\}$ is peaked.

Corollary 1. Let $X$ be a normal space, $\mathcal{C}$ a locally finite peaked open covering of $X$ with peaks $x_{C} \in C, E$ a Hausdorff topological vector space, and $f: X \rightarrow E$ a continuous function. Then the following are equivalent: 
(i) There exists a partition of unity $\left\{\varphi_{C}: C \in \mathcal{C}\right\}$ subordinate to $\mathcal{C}$ and vectors $a_{C} \in E$ such that

$$
f=\sum_{C \in \mathcal{C}} a_{C} \varphi_{C}
$$

(ii) For all $x \in X$,

$$
f(x) \in \operatorname{conv}\left\{f\left(x_{C}\right): x \in C \in \mathcal{C}\right\} .
$$

Proof. The points $x_{C} \in C$ being peaks we get $f\left(x_{C}\right)=a_{C}$ from (i). By Theorem 1, condition (i) implies that $f(x) \in \operatorname{conv}\left\{a_{C}: x \in C \in \mathcal{C}\right\}=$ $\operatorname{conv}\left\{f\left(x_{C}\right): x \in C \in \mathcal{C}\right\}$ for all $x \in X$. This proves the implication (i) $\Rightarrow$ (ii). The converse direction (ii) $\Rightarrow(\mathrm{i})$ can easily be seen by applying Theorem 1 to the vectors $a_{C}=f\left(x_{C}\right)$.

Let us point out that the normality of $X$ cannot be weakened in Theorem 1 as well as in Corollary 1 . In fact, the implications (ii) $\Rightarrow$ (i) from Theorem 1 and Corollary 1 can be used to characterize normal spaces among $\mathrm{T}_{1}$-spaces.

THEOREM 2. Let $X$ be a $\mathrm{T}_{1}$-space. Then the following are equivalent:

( $\alpha$ ) $X$ is normal.

( $\beta)$ The implication (ii) $\Rightarrow(\mathrm{i})$ from Theorem 1 holds true for all locally finite open coverings $\mathcal{C}$ of $X$, all Hausdorff topological vector spaces $E$, all continuous functions $f: X \rightarrow E$, and all vectors $a_{C} \in E, C \in \mathcal{C}$.

$(\gamma)$ The implication (ii) $\Rightarrow(\mathrm{i})$ from Corollary 1 holds true for all locally finite peaked open coverings $\mathcal{C}$ of $X$ with peaks $x_{C} \in C$, all Hausdorff topological vector spaces $E$, and all continuous functions $f: X \rightarrow E$.

The equivalences remain valid if $E$ is replaced by the particular space $\mathbb{R}$.

Proof. The implication $(\alpha) \Rightarrow(\beta)$ is given by Theorem $1 .(\beta) \Rightarrow(\gamma)$ was shown in the proof of Corollary 1 . To prove $(\gamma) \Rightarrow(\alpha)$, let $A, B \subseteq X$ be two disjoint closed non-empty sets. Applying Corollary 1 to $\mathcal{C}=\{X \backslash A, X \backslash B\}$, $E=\mathbb{R}$, and $f \equiv 1$ one obtains a partition function $\varphi=\varphi_{X \backslash B}$ such that $\left.\varphi\right|_{A} \equiv 1$ and $\left.\varphi\right|_{B} \equiv 0$.

We close this section with a corollary on partitions of unity which is well-known from the literature (cf. [10], p. 353). However, Theorem 1 gives rise to a very simple proof. One only has to apply the implication (ii) $\Rightarrow$ (i) to the constant function $f \equiv 1$ and the reals $a_{C}=1$.

Corollary 2. For any locally finite open covering $\mathcal{C}$ of a normal space $X$, there exists a partition of unity $\left\{\varphi_{C}: C \in \mathcal{C}\right\}$ on $X$ subordinate to $\mathcal{C}$.

2. A question from optimization theory. Another application of Theorem 1 concerns a question from optimization theory. We consider an $m$-dimensional polytope $P \subseteq \mathbb{R}^{m}$. A polytope is meant to be the convex hull 
of a finite set of points. The face lattice $\mathcal{F}(P)$ of $P$ is the set of all $k$-faces of $P,-1 \leq k \leq m$. A $k$-face of $P$ with $0 \leq k \leq m-1$ is a $k$-dimensional intersection of $P$ with a supporting hyperplane of $P$. Besides that, the empty set $\emptyset$ and the polytope $P$ itself are considered as the $(-1)$-face and the $m$-face of $P$, respectively. For example, the face lattice $\mathcal{F}(S)$ of a square $S \subseteq \mathbb{R}^{2}$ consists of $\emptyset$ (the $(-1)$-face), the four singletons $\{v\}$ formed by the vertices $v \in \operatorname{vert}(S)$ (the 0-faces), the four edges (the 1-faces), and $S$ (the 2-face). Note that all faces of a polytope $P$ are polytopes themselves.

The following simple fact is widely used in optimization theory: Let the continuous real-valued function $f \in C(P)$ be convex (resp. concave) and let $F \in \mathcal{F}(P)$ be a face of $P$. Then the restriction $\left.f\right|_{F}$ attains its maximum (resp. minimum) at a vertex of $F$.

We want to tackle a related problem: Given a polytope $P$, we ask for a characterization of all functions $f \in C(P)$ such that, for all faces $F \in \mathcal{F}(P)$, $\left.f\right|_{F}$ attains its minimum and its maximum at vertices of $F$, that is,

$$
\min f(F), \max f(F) \in f(\operatorname{vert}(F)) .
$$

Before giving the solution we generalize the problem in so far as we consider Euclidean complexes instead of single polytopes.

A Euclidean complex $\mathcal{P}$ in $\mathbb{R}^{m}$ is a non-empty collection of polytopes $P \subseteq \mathbb{R}^{m}$ satisfying the following three conditions (cf. [9], p. 313):

(C1) For all $P \in \mathcal{P}$, all faces of $P$ belong to $\mathcal{P}$ :

$$
P \in \mathcal{P} \Rightarrow \mathcal{F}(P) \subseteq \mathcal{P} .
$$

(C2) For all $P, Q \in \mathcal{P}$, the intersection $P \cap Q$ is a face of both $P$ and $Q$ :

$$
P, Q \in \mathcal{P} \Rightarrow P \cap Q \in \mathcal{F}(P) \cap \mathcal{F}(Q) .
$$

(C3) The system $\mathcal{P}$ is locally finite in the so-called underlying space

$$
|\mathcal{P}|=\bigcup_{P \in \mathcal{P}} P
$$

of $\mathcal{P}$. (Clearly, the topology of $|\mathcal{P}|$ is induced by the usual topology of $\mathbb{R}^{m}$.)

Euclidean complexes generalize the concept of a polytope in the sense that the face lattice of any polytope is a Euclidean complex. A useful reference concerning Euclidean complexes is [4], Chapter 2. Although the complexes considered there are supposed to be finite and the emphasis is even on the particular case where all polytopes are simplices, many arguments of that exposition apply to the general case.

The set $\operatorname{vert}(\mathcal{P})$ of the vertices of the complex $\mathcal{P}$ contains all vertices of polytopes from $\mathcal{P}$. The relative interior relint $(P)$ of a polytope $P$ is meant to be the interior of $P$ with respect to the topology of the affine space spanned 

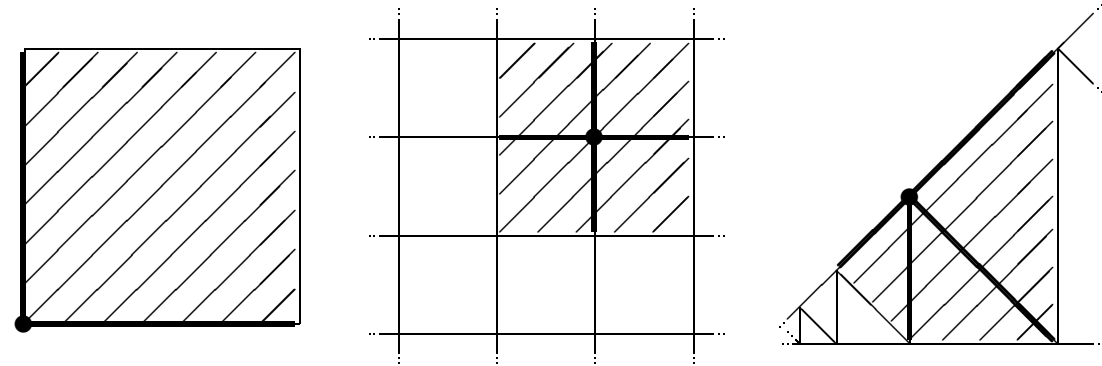

Fig. 1

by $P$. For a vertex $v \in \operatorname{vert}(\mathcal{P})$, the star of $v$ is defined by

$$
\operatorname{st}(v)=\bigcup_{P \in \mathcal{P}, v \in \operatorname{vert}(P)} \operatorname{relint}(P) .
$$

Examples of stars are illustrated in Figure 1.

Lemma. A Euclidean complex $\mathcal{P}$ in $\mathbb{R}^{m}$ has the following properties:

(a) The underlying space $|\mathcal{P}|$ can be expressed as the disjoint union

$$
|\mathcal{P}|=\bigcup_{P \in \mathcal{P}} \operatorname{relint}(P)
$$

(b) For all $P \in \mathcal{P}, v \in \operatorname{vert}(\mathcal{P})$, and $x \in|\mathcal{P}|$,

$$
x \in \operatorname{relint}(P) \Rightarrow(x \in \operatorname{st}(v) \Leftrightarrow v \in \operatorname{vert}(P)) .
$$

(c) The system $\{\operatorname{st}(v): v \in \operatorname{vert}(\mathcal{P})\}$ is a locally finite peaked open covering of the topological space $|\mathcal{P}|$ with peaks $v \in \operatorname{st}(v)$.

Proof. Part (a) can be obtained by following the proof of Theorem 2.2.2 from [4].

To prove (b), assume that $x \in \operatorname{relint}(P)$. First suppose that $x \in \operatorname{st}(v)=$ $\bigcup_{Q \in \mathcal{P}, v \in \operatorname{vert}(Q)} \operatorname{relint}(Q)$. Then, by (a), $P \in\{Q \in \mathcal{P}: v \in \operatorname{vert}(Q)\}$ and hence $v \in \operatorname{vert}(P)$. The opposite implication is a trivial consequence of the definition of $\operatorname{st}(v)$ and of the inclusion $x \in \operatorname{relint}(P)$.

Now we turn to (c). Applying the proofs of Assertions 2.3.12 and 2.3.15 from [4] one shows that $\{\operatorname{st}(v): v \in \operatorname{vert}(\mathcal{P})\}$ is an open covering of $|\mathcal{P}|$.

The peak property of a star st $(v)$ can be seen as follows: By (a), the only polytope $P \in \mathcal{P}$ with $v \in \operatorname{relint}(P)$ is $P=\{v\}$. By (b), we obtain $v \in \operatorname{st}(w)$ if and only if $w \in \operatorname{vert}(\{v\})$, i.e. $w=v$. Thus $v$ is a peak of $\operatorname{st}(v)$.

Finally, we fix a point $x \in|\mathcal{P}|$ in order to show that $\{\operatorname{st}(v): v \in \operatorname{vert}(\mathcal{P})\}$ is locally finite. By (C3), there exists a neighbourhood $U \subseteq|\mathcal{P}|$ of $x$ intersecting at most finitely many of the relatively open polytopes relint $(P)$, $P \in \mathcal{P}$. We assume that $U \cap \operatorname{relint}(P) \neq \emptyset$ for $P \in\left\{P_{1}, \ldots, P_{l}\right\}$ only. By (b), all the sets $\operatorname{st}(v)$ covering at least one point $y \in U$ must satisfy 
$v \in \operatorname{vert}\left(P_{1}\right) \cup \ldots \cup \operatorname{vert}\left(P_{l}\right)$. Accordingly, $U$ intersects only finitely many sets from $\{\operatorname{st}(v): v \in \operatorname{vert}(\mathcal{P})\}$.

Given a Euclidean complex $\mathcal{P}$, we have to characterize all functions $f \in$ $C(|\mathcal{P}|)$ with

$$
\min f(P), \max f(P) \in f(\operatorname{vert}(P)) \quad \text { for all } P \in \mathcal{P} \text {. }
$$

These functions turn out to be linear combinations of partitions of unity subordinate to the covering $\{\operatorname{st}(v): v \in \operatorname{vert}(\mathcal{P})\}$.

TheOREM 3. Let $\mathcal{P}$ be a Euclidean complex in $\mathbb{R}^{m}$ and let $f \in C(|\mathcal{P}|)$. Then the following are equivalent:

(i) The function $f$ can be represented as

$$
f=\sum_{v \in \operatorname{vert}(\mathcal{P})} \lambda_{v} \varphi_{v}
$$

with coefficients $\lambda_{v} \in \mathbb{R}$ and a partition of unity $\left\{\varphi_{v}: v \in \operatorname{vert}(\mathcal{P})\right\}$ on $|\mathcal{P}|$ subordinate to the covering $\{\operatorname{st}(v): v \in \operatorname{vert}(\mathcal{P})\}$.

(ii) For all polytopes $P \in \mathcal{P}$,

$$
\min f(P), \max f(P) \in f(\operatorname{vert}(P)) .
$$

Proof. The above preparations make the proof rather easy, although the implication from the global condition (i) to the more or less local one (ii) is not as trivial as in Theorem 1. We apply Corollary 1 to the locally finite peaked open covering $\{\operatorname{st}(v): v \in \operatorname{vert}(\mathcal{P})\}$ and the peaks $v \in \operatorname{st}(v)$. Accordingly, condition (i) is equivalent to

$$
\forall x \in|\mathcal{P}|: f(x) \in \operatorname{conv}\{f(v): x \in \operatorname{st}(v)\} .
$$

The arguments we shall give below show that the following six claims are equivalent to (i) as well:

(4) $\forall x \in|\mathcal{P}|, \forall P \in \mathcal{P}: x \in \operatorname{relint}(P) \Rightarrow f(x) \in \operatorname{conv}(f(\operatorname{vert}(P)))$,

(5) $\forall x \in|\mathcal{P}|, \forall P \in \mathcal{P}: x \in P \Rightarrow f(x) \in \operatorname{conv}(f(\operatorname{vert}(P)))$,

(6) $\forall P \in \mathcal{P}: f(P) \subseteq \operatorname{conv}(f(\operatorname{vert}(P)))$,

(7) $\forall P \in \mathcal{P}: \min f(P), \max f(P) \in \operatorname{conv}(f(\operatorname{vert}(P)))$,

(8) $\forall P \in \mathcal{P}: \min f(P), \max f(P) \in f(\operatorname{vert}(P))$.

Indeed, $(2) \Leftrightarrow(3)$ and $(3) \Leftrightarrow(4)$ are consequences of claims (a) and (b), respectively, from the lemma. $(4) \Leftrightarrow(5)$ is due to the continuity of $f$. The equivalences $(5) \Leftrightarrow(6)$ and $(6) \Leftrightarrow(7)$ are obvious. The final step $(7) \Leftrightarrow(8)$ is based on the trivial inclusion $\operatorname{vert}(P) \subseteq P$.

Hence the assertion $(\mathrm{i}) \Leftrightarrow(\mathrm{ii})$ is shown, since (8) coincides with (ii). 
3. An approximation class on $\left([-1,1]^{m}, d_{\infty}\right)$. In this section we shall sketch the approximation by so-called controllable partitions of unity (see [11], [8], [7]). This method of approximating real-valued continuous functions on compact metric spaces gave rise to the fairly general considerations of the present paper. Theorem 3 will be applied to describe a particular approximation class on the cube $\left([-1,1]^{m}, d_{\infty}\right)$.

Let $(X, d)$ be a compact metric space. Then the kth entropy number of a subset $S \subseteq X$ is given by

$$
\varepsilon_{k}(S)=\inf \{\varepsilon>0: S \text { can be covered by } k \text { balls of radius } \varepsilon\}
$$

(cf. [3], p. 7). A finite partition of unity $\left\{\varphi_{1}, \ldots, \varphi_{k}\right\}$ on $X$ is called controllable if either $k=1$, i.e. $\left\{\varphi_{1}\right\}=\left\{\mathbf{1}_{X}\right\}$ is the trivial partition of unity, or the partition satisfies the geometric uniformity condition

$$
\varepsilon_{1}\left(\operatorname{supp}\left(\varphi_{i}\right)\right)<\varepsilon_{k-1}(X) \quad \text { for } 1 \leq i \leq k
$$

restricting the size of the supports $\operatorname{supp}\left(\varphi_{i}\right)=\overline{\left\{x \in X: \varphi_{i}(x) \neq 0\right\}}$ of the single functions $\varphi_{i}$. One uses linear combinations of controllable partitions of unity from the classes

$$
\begin{aligned}
\Phi_{n}(X)=\left\{\sum_{i=1}^{k} \lambda_{i} \varphi_{i}:\right. & \lambda_{i} \in \mathbb{R},\left\{\varphi_{1}, \ldots, \varphi_{k}\right\} \text { is a controllable } \\
& \text { partition of unity on } X \text { with cardinality } k \leq n\},
\end{aligned}
$$

$n=1,2, \ldots$, in order to define a sequence of approximation numbers $a_{n}(f)$ for functions $f \in C(X)$ by

$$
a_{n}(f)=\inf \left\{\|f-\varphi\|: \varphi \in \Phi_{n}(X)\right\},
$$

$\|\cdot\|$ denoting the maximum norm of $C(X)$. These approximation quantities give rise to the Jackson type estimate

$$
a_{n}(f) \leq \omega\left(f, \varepsilon_{n}(X)\right)
$$

for all $f \in C(X)$ and $n \in\{1,2, \ldots\}$, where $\omega(f, \delta)=\sup \{|f(x)-f(y)|$ : $d(x, y) \leq \delta\}$ is the modulus of continuity.

Now we turn to approximation on the cube $[-1,1]^{m}$ equipped with the maximum metric $d_{\infty}$. In [11] it is shown that controllable partitions of unity of cardinality $k \geq 2$ on a space $X$ exist if and only if the jump condition $\varepsilon_{k}(X)<\varepsilon_{k-1}(X)$ is satisfied. The cube $[-1,1]^{m}$ has the entropy numbers $\varepsilon_{l^{m}}\left([-1,1]^{m}\right)=\varepsilon_{l^{m}+1}\left([-1,1]^{m}\right)=\ldots=\varepsilon_{(l+1)^{m}-1}\left([-1,1]^{m}\right)=1 / l$ for $l \geq 1$ (cf. [1], [2]). Thus there exist controllable partitions of unity exactly for the cardinalities $k=1,2^{m}, 3^{m}, \ldots$ In particular, the approximation classes $\Phi_{n}\left([-1,1]^{m}\right), 1 \leq n \leq 2^{m}-1$, coincide and consist of the constant functions only. The first interesting class is $\Phi_{2^{m}}\left([-1,1]^{m}\right)$ formed by all linear combinations of controllable partitions of unity of cardinality $2^{m}$. We shall characterize all functions $f \in C\left([-1,1]^{m}\right)$ belonging to the closure $\overline{\Phi_{2^{m}}\left([-1,1]^{m}\right)}$, 
which means that $a_{2^{m}}(f)=0$. In [8] this characterization is already given for the one-dimensional case $m=1$.

THEOREM 4. Let $f$ be a continuous real-valued function on the compact metric space $\left([-1,1]^{m}, d_{\infty}\right)$. Then the following are equivalent:

(i) $f \in \overline{\Phi_{2^{m}}\left([-1,1]^{m}\right)}$.

(ii) For all faces $Q \in \mathcal{F}\left([-1,1]^{m}\right)$,

$$
\min f(Q), \max f(Q) \in f(\operatorname{vert}(Q)) \text {. }
$$

Proof. First we point out that a partition of unity $\Phi$ of cardinality $2^{m}$ on $[-1,1]^{m}$ is controllable if and only if there exists some $\varepsilon>0$ such that the partition has the structure

$$
\Phi=\left\{\varphi_{v}: v \in \operatorname{vert}\left([-1,1]^{m}\right)\right\} \quad \text { with } \operatorname{supp}\left(\varphi_{v}\right) \subseteq \overline{B_{2-\varepsilon}(v)}
$$

$B_{2-\varepsilon}(v)$ denoting the open ball of radius $2-\varepsilon$ centred at $v$. Indeed, $\Phi$ must satisfy the uniformity condition $\varepsilon_{1}(\operatorname{supp}(\varphi))<\varepsilon_{2^{m}-1}\left([-1,1]^{m}\right)=1$ for all $\varphi \in \Phi$. That is, there exists some $\varepsilon>0$ such that $\varepsilon_{1}(\operatorname{supp}(\varphi)) \leq 1-\varepsilon / 2$ or, in other words, each $\operatorname{support} \operatorname{supp}(\varphi)$ is contained in a closed subcube with edges of length $2-\varepsilon$. Thus each vertex $v \in[-1,1]^{m}$ belongs to exactly one of the $2^{m}$ supports, say $v \in \operatorname{supp}\left(\varphi_{v}\right)$ and $\Phi=\left\{\varphi_{v}: v \in \operatorname{vert}\left([-1,1]^{m}\right)\right\}$. The condition of being contained in a cube with edges of length $2-\varepsilon$ amounts to $\operatorname{supp}\left(\varphi_{v}\right) \subseteq \overline{B_{2-\varepsilon}(v)}$ for $v \in \operatorname{vert}\left([-1,1]^{m}\right)$.

Next we observe that the covering $\mathcal{C}=\left\{\operatorname{st}(v): v \in \operatorname{vert}\left([-1,1]^{m}\right)\right\}$ of the cube $[-1,1]^{m}$ induced by the face lattice $\mathcal{F}\left([-1,1]^{m}\right)$ in the sense of the previous section is given by $\operatorname{st}(v)=B_{2}(v), v \in \operatorname{vert}\left([-1,1]^{m}\right)$. Hence a partition of unity $\left\{\varphi_{v}: v \in \operatorname{vert}\left([-1,1]^{m}\right)\right\}$ is subordinate to $\mathcal{C}$ if and only if

$$
\varphi_{v}^{-1}((0,1]) \subseteq B_{2}(v) \quad \text { for } v \in \operatorname{vert}\left([-1,1]^{m}\right) .
$$

Now let us verify the implication (i) $\Rightarrow($ ii). We have already seen that any controllable partition of unity of cardinality $2^{m}$ is subordinate to $\mathcal{C}$. Hence, by Theorem 3 , as any function $\varphi \in \Phi_{2^{m}}\left([-1,1]^{m}\right)$ is a linear combination of a partition of unity of this kind, it satisfies the extremality condition

$$
\min \varphi(Q), \max \varphi(Q) \in \varphi(\operatorname{vert}(Q)) \quad \text { for all } Q \in \mathcal{F}\left([-1,1]^{m}\right) .
$$

It can easily be checked that this remains true if one passes from elements $\varphi$ of the class $\Phi_{2^{m}}\left([-1,1]^{m}\right)$ to functions from the closure $\overline{\Phi_{2^{m}}\left([-1,1]^{m}\right)}$.

The proof of the converse direction (ii) $\Rightarrow(\mathrm{i})$ again uses Theorem 3 . We assume that $f$ satisfies condition (ii). Then it must admit a representation

$$
f=\sum_{v \in \operatorname{vert}\left([-1,1]^{m}\right)} \lambda_{v} \varphi_{v}
$$

with $\lambda_{v} \in \mathbb{R}$ and a partition of unity $\left\{\varphi_{v}: v \in \operatorname{vert}\left([-1,1]^{m}\right)\right\}$ subordinate to $\mathcal{C}$, i.e. $\varphi_{v}^{-1}((0,1]) \subseteq B_{2}(v)$. Let $0<\varepsilon<1$. We consider the metric 
projection $P_{\varepsilon}:[-1,1]^{m} \rightarrow[-1+\varepsilon, 1-\varepsilon]^{m}$ given by

$$
P_{\varepsilon}(x)=y \Leftrightarrow d_{2}(x, y)=\min \left\{d_{2}(x, z): z \in[-1+\varepsilon, 1-\varepsilon]^{m}\right\},
$$

$d_{2}$ denoting the Euclidean distance. Moreover, let $D_{\varepsilon}:[-1+\varepsilon, 1-\varepsilon]^{m} \rightarrow$ $[-1,1]^{m}$ be the dilatation mapping $y$ onto $y /(1-\varepsilon)$. Now a new partition of unity $\left\{\varphi_{v}^{(\varepsilon)}: v \in \operatorname{vert}\left([-1,1]^{m}\right)\right\}$ can be defined by

$$
\varphi_{v}^{(\varepsilon)}=\varphi_{v} D_{\varepsilon} P_{\varepsilon} \quad \text { for } v \in \operatorname{vert}\left([-1,1]^{m}\right) \text {. }
$$

Then obviously

$$
\begin{aligned}
\operatorname{supp}\left(\varphi_{v}^{(\varepsilon)}\right) & =\overline{P_{\varepsilon}^{-1} D_{\varepsilon}^{-1}\left(\varphi_{v}^{-1}((0,1])\right)} \subseteq \overline{P_{\varepsilon}^{-1} D_{\varepsilon}^{-1}\left(B_{2}(v)\right)} \\
& =\overline{P_{\varepsilon}^{-1}\left([-1+\varepsilon, 1-\varepsilon]^{m} \cap B_{2-\varepsilon}(v)\right)}=\overline{B_{2-\varepsilon}(v)}
\end{aligned}
$$

Therefore the partition of unity $\left\{\varphi_{v}^{(\varepsilon)}: v \in \operatorname{vert}\left([-1,1]^{m}\right)\right\}$ is controllable and $\varphi_{\varepsilon}=\sum_{v \in \operatorname{vert}\left([-1,1]^{m}\right)} \lambda_{v} \varphi_{v}^{(\varepsilon)}$ belongs to $\Phi_{2^{m}}\left([-1,1]^{m}\right)$. We obtain $\varphi_{\varepsilon}=$ $f D_{\varepsilon} P_{\varepsilon}$ and

$$
\left\|f-\varphi_{\varepsilon}\right\| \leq \omega(f, 2 \varepsilon)
$$

since $d_{\infty}\left(x, D_{\varepsilon} P_{\varepsilon}(x)\right) \leq d_{\infty}\left(x, P_{\varepsilon}(x)\right)+d_{\infty}\left(P_{\varepsilon}(x), D_{\varepsilon} P_{\varepsilon}(x)\right) \leq \varepsilon+\varepsilon=2 \varepsilon$ for $x \in[-1,1]^{m}$.

Consequently, $f$ can be written as a limit $f=\lim _{\varepsilon \downarrow 0} \varphi_{\varepsilon}$ of functions $\varphi_{\varepsilon} \in \Phi_{2^{m}}\left([-1,1]^{m}\right)$. This is our claim.

One can read Theorem 4 as an approximation-theoretic characterization of extremal functions on the cube. Besides that, Theorem 4 shows that the first non-trivial approximation class $\Phi_{2^{m}}\left([-1,1]^{m}\right)$ on the cube is surprisingly large and serves as an example of a non-linear approximation class.

Note that Theorem 4 is essentially based on the choice of the metric $d_{\infty}$ on the cube, which reflects the polyhedral structure of $[-1,1]^{m}$. We conjecture that an analogue of the above theorem can be proved for all polytopes $P$. Then the extremal functions $f \in C(P)$ would arise as the functions with $a_{k}(f)=0$ where $k$ denotes the number of vertices of $P$. The crucial step to such a generalization of Theorem 4 seems to be the definition of an appropriate metric $d_{P}$ on $P$. For instance, if $P$ is the unit ball of a Banach space and if $d_{\|\cdot\|}$ is the metric on $P$ induced by the norm, then the above-mentioned analogue of Theorem 4 need not be true for the compact space $\left(P, d_{\|\cdot\|}\right)$ in general.

Acknowledgments. The author wishes to express his gratitude to the unknown referee for an essential advice, which has led to a shorter and more general proof of Theorem 1. Moreover, he thanks Irmtraud Stephani for stimulating discussions concerning the presentation of the above results. 


\section{References}

[1] V. F. Babenko and S. A. Pichugov, Approximation of continuous vector functions, Ukrainian Math. J. 46 (1994), 1585-1599.

[2] W. Börner and C. Richter, Entropy, capacity and arrangements on the cube, Beiträge Algebra Geom. 38 (1997), 227-232.

[3] B. Carl and I. Stephani, Entropy, Compactness and the Approximation of Operators, Cambridge Tracts in Math. 98, Cambridge Univ. Press, 1990.

[4] R. Engelking and K. Sieklucki, Topology. A Geometric Approach, Sigma Ser. Pure Math. 4, Heldermann, Berlin, 1992.

[5] E. Michael, Continuous selections. I, Ann. of Math. 63 (1956), 361-382.

[6] E. Michael and A. Pełczyński, Peaked partition subspaces of $C(X)$, Illinois J. Math. 11 (1967), 555-562.

[7] C. Richter, A chain of controllable partitions of unity on the cube and the approximation of Hölder continuous functions, ibid. 43 (1999), 159-191.

[8] C. Richter and I. Stephani, Entropy and the approximation of bounded functions and operators, Arch. Math. (Basel) 67 (1996), 478-492.

[9] W. Rinow, Lehrbuch der Topologie, Deutscher Verlag Wiss., Berlin, 1975.

[10] Z. Semadeni, Banach Spaces of Continuous Functions I, PWN-Polish Scientific Publ., Warszawa, 1971.

[11] I. Stephani, Entropy and the approximation of continuous functions, Arch. Math. (Basel) 58 (1992), 280-287.

Equipe d'Analyse

Université Paris VI

Case 186

4, Place Jussieu

75252 Paris Cedex 05, France

E-mail: richterc@minet.uni-jena.de

Received November 22, 1999

Revised version May 16, 2002 